\title{
RELIGION NATURELLE ET SCEPTICISME RELIGIEUX
}

\author{
Jacqueline LAGRÉE
}

Résumé: Dans son livre sur l'Histoire du scepticisme d'Érasme à Spinoza, Richard H. Popkin présente la théorie de la vérité proposée par Edward Herbert de Cherbury dans le De veritate comme une machine de guerre lourde et impuissante dirigée contre le scepticisme religieux de l'âge classique. L'article entend montrer que le lourd dispositif conceptuel du De veritate ne constitue pas le cœur de la réponse cherburienne au scepticisme mais seulement le fondement théorique d'un perspectivisme religieux immédiatement corrigé par la défense d'un credo minimum stable et universel. Par là, Cherbury accomplit le programme du pragmatisme religieux de Sextus Empiricus, en recherchant non pas la religion la plus vraie mais la meilleure pour permettre aux hommes de vivre en paix dans le respect mutuel de leurs croyances.

Mots-cLés : Richard H. Popkin, Cherbury, religion naturelle, vérité, scepticisme, perspectivisme.

ABSTRACT: In the History of scepticism from Erasmus to Spinoza, Richard H. Popkin presents Edward Herbert of Cherbury's theory of truth as a heavy and unsuccessful weapon against the religious scepticism of the seventeenth century. This paper claims to show that this heavy conceptual device is not the very heart of the Cherburian response to scepticism but precisely the theoretical foundation of a religious perspectivism which is immediately corrected by a minimal credo which is universal and stable. In doing so, Cherbury fulfills the religious pragmativism's program of Sextus Empiricus for he does not try to find the truest religion but the best one, the one which allows men to live in peace, in a mutual respect of their beliefs.

KEYWORDS : Richard H. Popkin, Cherbury, natural religion, truth, scepticism, perspectivism. Revue de synthèse : $4^{\text {e }}$ S. $n^{\circ \mathrm{s}}$ 2-3, avr.-sept. 1998, p. 257-269. 
Zusammenfassung : In seinem Buch über Die Geschichte des Skeptizismus von Erasmus bis Spinoza präsentiert Richard H. Popkin die Edward Herbert von Cherbury in De veritate vorgestellte Theorie der Wahrheit als eine schwerfällige und wirkungslose Waffe im Kampf gegen den religiösen Skeptizismus des 17. Jahrhunderts. In diesem Artikel wird gezeigt, daß die umständliche Argumentation in De veritate nicht den Kern von Cherburys Antwort auf den Skeptizismus darstellt, sondern nur die theoretische Begründung eines religiösen Perspektivismus bildet, der unmittelbar darauf durch die Verteidigung eines minimalen, allgemeingültigen und unumstößlichen Kredo korrigiert wird. Auf diese Weise vollendet Cherbury das auf Sextus Empiricus zurückgehende Programm eines religiösen Pragmatismus, indem er nicht nach der Religion mit dem größten Wahrheitsgehalt fragt, sondern nach derjenigen Religion, die am besten dazu geeignet ist, den Menschen ein friedliches Leben auf der Grundlage eines gegenseitigen Respekts der Glaubensüberzeugungen zu ermöglichen.

STICHWörter : Richard H. Popkin, Cherbury, Naturreligion, Wahrheit, Skeptizismus, Perspektivismus.

Jacqueline LAGRÉE, née en 1947, est professeur de philosophie et directrice de l'UFR de philosophie de l'université de Rennes I. Ses recherches portent sur le spinozisme, le néo-stoïcisme, la religion naturelle.

Adresse : UFR de philosophie, Campus de Beaulieu, F-35042 Rennes Cedex.

Courrier électronique : jacqueline-lagree@univ-rennes1.fr 
Le problème qui préoccupe Richard $\mathrm{H}$. Popkin dans son livre Histoire $d u$ scepticisme d'Érasme à Spinoza n'est pas celui de la religion naturelle mais plutôt celui du sens et des effets religieux du scepticisme au xvII ${ }^{e}$ siècle. Eut-il pour effet un fidéisme religieux plus favorable finalement au catholicisme qu'au protestantisme, comme chez Montaigne, ou bien conduisit-il à terme - du moins est-ce ainsi qu'il fut perçu chez leurs adversaires - à l'incroyance hautaine par laquelle on caractérise souvent les libertins? Il est clair que les sympathies et les arguments de Popkin vont dans le sens de la première réponse : « $\mathrm{J}$ 'ai en fait plus de sympathie pour ceux qui employèrent les idées sceptiques et fidéistes des nouveaux pyrrhoniens à des fins religieuses plutôt que séculières '. " Ce fut l'un de ses mérites, et non des moindres, d'avoir souligné l'existence d'un scepticisme à tonalité religieuse à l'âge classique.

Pourquoi donc la religion naturelle? Parce qu'elle constitue chez Edward Herbert de Cherbury une des réponses constructives à l'entreprise de sape des croyances qui résulte de la mise en cuvre du dixième trope sur la relativité des mœurs et des convictions. Popkin fut l'un des premiers ${ }^{2}$ à prendre au sérieux la philosophie de la religion proposée par Cherbury sans réduire celui-ci, comme tant d'autres l'avaient fait aux siècles précédents, à un pur précurseur du déisme.

Certes, il y eut bien auparavant le travail monumental de Mario Rossi, La Vita, le opere i tempi di Eduardo Di Cherbury ${ }^{3}$. Mais Rossi écrit après la guerre, avec des intentions idéologiques déclarées : Cherbury est, avec Thomas Hobbes, une des Sources du déisme et du matérialisme moderne ${ }^{4}$ qui sont, selon lui, la source intellectuelle des malheurs qui affectent l'Europe. Comme on voit, la perspective idéologique et politique interfère lourdement avec le travail d'érudition incontestablement très sérieux. Il nous faut donc examiner les présupposés, la pertinence et éventuellement, les limites de la lecture de Cherbury par Popkin.

1. Richard H. PoPKIN, Histoire du scepticisme d'Érasme à Spinoza, trad. Christine Hivet, présentation Catherine LaRRĖRe, Paris, Presses universitaires de France (Léviathan), 1995, p. 31 (cité par la suite comme $H S$ ).

2. Avant Richard BEDFORD, The Defense of truth, Herbert of Cherbury and the 17th century, Manchester, Manchester University Press, 1979; Jacqueline LAGRÉE, Le Salut du laïc : Herbert de Cherbury, Paris, Vrin, 1989.

3. Mario Rossı, La Vita, le opere $i$ tempi di Eduardo Di Cherbury, 3 vol., Florence, 1947.

4. ID., Alle fonti del deismo e del materialismo moderno, Florence, 1942. 
LE CONTEXTE DE L'EXAMEN

Popkin analyse la philosophie de Cherbury en se limitant à sa théorie de la vérité présentée dans le $D e$ veritate et en négligeant complètement l'appendice (sur les notions communes religieuses) et le traité annexe ( $L a$ Religion du laïc), tout comme il ne dit mot du gros traité de religion comparée, inspiré de Gérard-Jean Vossius et John Selden, intitulé De religione gentilium (1663). C'est dire qu'il prend Cherbury pour un épistémologue ou un philosophe soucieux, comme Descartes, de fonder le système du savoir sur une vérité inébranlable, la théorie des notions communes étant censée jouer pour Cherbury, selon Popkin, le rôle crucial du cogito dans la métaphysique cartésienne. C'est là une grave erreur de perspective, à mon sens, car l'essentiel de la pensée cherburienne ne réside pas dans ses zététiques - si mal nommées car il est fort peu probable qu'elles aient jamais servi à trouver quelque chose - mais bien dans l'application pratique de la théorie des notions communes à la question du conflit religieux.

Or le contexte initial dans lequel Popkin situe la crise sceptique à la Renaissance éclaire bien la démarche de Cherbury, surtout si on la rapproche de celle de Sébastien Castellion ${ }^{5}$. Comme Castellion en un sens, l'ceuvre de Cherbury constitue un « art de douter et de croire, d'ignorer et de savoir ». C'est en ayant établi (ou en croyant l'avoir fait) ce que l'on peut savoir et ce qu'il convient d'ignorer - puisqu'on ne dispose pas des conditions nécessaires pour atteindre en ce domaine une vérité définitivement assurée - qu'on garantira la paix civile et l'harmonie entre des hormmes qui pensent différemment de choses qui, de toute façon, excèdent les capacités humaines de connaissance en cette vie. Car Cherbury ne doute pas que, de même que nos capacités perceptives et cognitives dépassent largement celles du fœetus ou du nourrisson que nous fûmes, nos capacités à venir, lorsque nous aurons atteint la vision béatifique, nous permettront de connaître ce qu'actuellement nous ignorons et de comprendre ce qui demeure confus et embrouillé en cette vie.

«Il faut donc avoir ici un peu de patience jusqu'à ce que nous soyons hors de ce corps et de ce monde ${ }^{6}$. »

5. $H S$, p. $44-45$.

6. De veritate, Paris, 1624 (cité $D V$ avec p. édition latine suivie de la p. de la traduction française par MERSENNE de 1639), 27/36. 
L'ANALYSE DE POPKIN

Popkin reproche à Cherbury de ne «pas avoir compris le problème essentiel ». Mais quel est ce problème essentiel et est-ce bien le même pour Popkin et pour Cherbury? Pour Popkin, le scepticisme suscite à la fois une crise théologique (qui dit la vérité sur l'Écriture sainte? est-ce le magistère et la tradition ou bien l'inspiration de l'Esprit saint parlant au cœur du fidèle?) et une crise du savoir : l'aristotélisme triomphant est menacé par les avancées de la science nouvelle (Copernic, Galilée) et la crise sceptique vient ébranler dangereusement la confiance dans les critères traditionnels de la vérité : si une science établie depuis tant de siècles se voit si brutalement contestée, à qui se fier? Le défi essentiel lancé par le renouveau du scepticisme, et auquel il faut de toute urgence répondre, est donc quasi uniquement le problème métaphysique du fondement de la vérité et le problème épistémologique du critère de la vérité.

Popkin envisage ainsi trois types ${ }^{7}$ de réponses philosophiques à partir de $1624^{8}:$ a) les réfutations fondées sur les principes de la philosophie aristotélicienne (par exemple, les jésuites); b) les réfutations qui reconnaissaient la force et la valeur des arguments pyrrhoniens avant de tenter de modérer les effets du scepticisme absolu (Gassendi); c) les réfutations qui visaient à construire un nouveau système philosophique afin de répondre au défi lancé par le scepticisme (Descartes, Spinoza). Dans quel type faudrait-il ranger Cherbury? Dans le premier? Certes, Popkin a tendance à faire de Cherbury un néo-aristotélicien mais cela ne convient pas, à la fois parce que l'horizon intellectuel antique de Cherbury n'est pas le Lycée mais le Portique et parce que la visée cherburienne n'est pas épistémologique mais essentiellement pratique.

Pour Cherbury, le problème essentiel de son époque, c'est celui de la liberté de penser et de croire du laiic et des moyens d'éviter la haine théologique, d'accéder au mieux à la paix en ce monde et à la béatitude dans l'autre, sans pour autant tomber dans l'indécision et le doute généralisé. Dans un cas l'adversaire, c'est le scepticisme qui ruine la vérité, dans l'autre c'est le dogmatisme qui enchaîne la liberté. La première perspective est théorique (d'où les précautions pratiques du Discours de la méthode);

7. $H S$, p. 167.

8. 1624 n'est pas seulement la date de la première publication de Gassendi mais aussi celle, ou presque, du $D V$ de Cherbury et du Droit de la guerre et de la paix de Grotius, ce qui constitue trois axes de développement d'un nouveau naturalisme : scientifique (Galilée, la nature est écrite en langage mathématique), juridique (Grotius) et religieux (Cherbury). 
la seconde est uniquement pratique ou elle n'est théorique, pour un temps, qu'à des fins pratiques.

Popkin centre toute sa présentation de Cherbury ${ }^{9}$ sur la théorie cherburienne de la vérité :

« Herbert avançait que l'homme était capable de savoir quelque chose. Ce qu'il nous fallait pour connaître et évaluer notre savoir, c'était une définition de la vérité, un critère de la vérité et une méthode permettant de découvrir la vérité. Une fois en leur possession, nous n'accorderions plus aucun crédit au scepticisme car nous comprendrions alors qu'il existait certaines conditions dans lesquelles nos facultés étaient capables de connaître les objets ${ }^{10}$."

Avant d'élargir et, par là même, de critiquer la perspective adoptée par Popkin, voyons donc ce qu'il en est de l'épistémologie cherburienne.

\section{La théorie de la vérité et sa critique}

Cherbury distingue quatre types de vérité auxquelles correspondent des types et des conditions de conformité particulières :

a) la veritas rei ou vérité de la chose en elle-même, c'est-à-dire la vérité dont l'atteinte est précisément niée par l'école sceptique; il y a convertibilité à ce niveau de la vérité et de l'être (ens).

b) la veritas apparentiae ou vérité de l'apparaître de la chose, vérité du phénomène, celle-là même et la seule que reconnaissent les sceptiques. Elle est toujours vraie en ce sens que lorsque, malade, je perçois amer le miel qui est doux, il est vrai que je le goute comme amer. Il faut done distinguer la vérité de l'apparaître (le miel m'apparaît amer) et la vérité de la chose (le miel est doux); la fausse apparence a sa vérité mais cette vérité est conditionnelle, c'est-à-dire relative à la situation, aux conditions d'aperception, à l'état des sens de celui qui perçoit. Pour passer légitimement de la vérité de l'apparaître à la vérité de la chose, il faut donc vérifier l'état des organes d'appréhension (les sens externes ou le sens interne) et les conditions de conformité de l'organe à l'objet, donc passer par deux médiations qui sont la vérité du concept et la vérité de l'entendement.

c) la veritas conceptus, vérité du concept ou vérité de notre représentation, désigne la conception de la chose, laquelle est fonction de notre situation et des limites de nos facultés de connaître mais ne constitue pas encore la vérité objective.

d) la veritas intellectus, la vérité de l'entendement désigne l'instance judicatoire ultime, celle qui juge des vérités inférieures (du phénomène et de la représentation intellectuelle) par les notions communes universelles.

9. La moitié d'un chapitre sur dix, soit 12 pages sur 314 , donc finalement for peu.

10. $H S$, p. 204. 


\section{Les conditions de conformation}

Pour obtenir une vérité, il faut mettre en relation un sujet doté d'un certain nombre de facultés, un objet et une faculté adéquate à la saisie de cet objet. Mais il faut, en outre, que la relation du sujet à l'objet, via la faculté, se fasse dans des conditions déterminées de conformité qui varient selon le niveau de vérité envisagé. On peut donc dire que Herbert prend en compte les arguments sceptiques pour assumer la relativité de la connaissance, ou plus exactement son caractère perspectiviste, ce qui ne détruit pas la connaissance comme telle dès lors que la perspective est reconnue comme perspective et qu'on en connaît les lois. Popkin critique tout particulièrement ces analyses cherburiennes car elles font référence à l'homme normal ou sain d'esprit, ce qui implique un critère de normalité qui est justement ce qui est mis en question par le scepticisme. Les conditions de conformation sont :

a) pour la vérité de l'objet, qu'il ait quelque analogie (rapport) avec nous : par exemple, nous sommes incapables de percevoir le très petit (l'atome, une odeur très subtile) ou le très grand; surtout, nous ne concevons l'infini et l'éternel que sub ratione fini et temporis".

b) pour la vérité de l'apparaitre : un certain laps de temps, un milieu adéquat de perception, une bonne distance d'aperception, et choses semblables qui impliquent une conception perspectiviste de la perception, susceptible d'être corrigée dans ses illusions par la connaissance de la vérité d'entendement.

c) pour la vérité du concept ou de notre représentation mentale de la chose : une bonne qualité des facultés d'appréhension, une application adéquate à l'objet (attention, application des bonnes qualités aux bons objets, par exemple le sens interne ou conscience ${ }^{12}$ pour les réalités invisibles, les sens externes pour les visibles) :

«Les facultés ne se peuvent pas bien conformer qu'avec leurs propres objets, avec lesquels ils ont du rapport et de l'affinité. C'est pourquoi la pensée ne comprend l'infini que sous l'ombre du fini (nisi finite a conceptu) ni l'éternel que sous la raison du temps; quoique nous ayons des facultés intérieures qui répondent à l'infini \& à l'éternel, à savoir celles qui sont éternelles \& lesquelles nous désirons demeurer éternellement. Et néanmoins, l'entendement assorti de ses communes notions enseigne que l'infini surpasse notre pensée \& que l'éternel n'est pas mesuré par notre temps. Il faut donc avoir ici un peu de patience jusqu'à ce que nous soyons hors de ce corps et de ce monde. Au reste il semble que nous soyons trop subtils dans les choses grossières \& que nous soyons trop grossiers dans les subtiles ${ }^{13}$. »

11. $D V, 14 / 19$.

12. La conscience qui comprend la conscience morale, le désir de béatitude, l'espérance, la foi, le sentiment de la liberté, le sentiment de l'existence de Dieu. Voir $D V, 82 / 109$ sq.

13. $D V, 27 / 36$; voir aussi, 14/19. 
d) Pour la vérité de l'entendement, vérité la plus décisive puisqu'elle sert de critère et de moyen de contrôle aux vérités précédentes, les conditions de conformation consistent dans la conformité avec les notions communes qui sont les principes des sciences, la source de toute recherche.

"Les vérités de l'entendement sont certaines notions communes qui sont en tous ceux qui sont sains et entiers lesquelles, comme parties des sciences et puisées de la sagesse universelle, sont gravées et empreintes dans l'intérieur par le dictamen de la nature ${ }^{14}$."

Mais contrairement à ce qu'écrit Popkin à plusieurs reprises, ici manifestement influencé par la lecture de Locke, les notions communes cherburiennes sont, exactement comme chez les stoïciens, des notions historiquement construites de la même façon par tous les êtres raisonnables (à partir de 7 ans, l'âge de raison), ce ne sont pas des notions innées. Qu'elles soient gravées et empreintes en nous ne signifie pas qu'elles le soient à la naissance mais que c'est par la nature et non par l'enseignement et la culture qu'elles le sont.

Cherbury est ici visiblement influencé par Grotius qui est de ses amis et qui, dans les mêmes années exactement, rédige son Droit de la guerre et de la paix. Or, parmi les notions communes, Cherbury ne cite pas seulement les notions communes religieuses, elles-mêmes citées par Grotius en plein cœur du Droit de la guerre et de la paix, mais également les notions communes juridiques (les préceptes d'Ulpien) et la règle d'or ${ }^{15}$ ou bien encore des pulsions naturelles (des hormaï, pour reprendre le vocable stoïcien correspondant exactement) telles que le souci de sa conservation ou bien le désir de béatitude. En outre, ces notions sont moins factuelles que de droit : tout le monde s'accorde, doit s'accorder et donc s'accordera ${ }^{16}$ sur elles car elles sont rationnelles et portent l'empreinte de la nature qui résiste à toutes les déformations culturelles et à tous les préjugés. Popkin voit dans la pensée de Cherbury un aristotélisme corrigé; il ne voit pas le caractère fortement stoïcien de cette conception.

\section{Essai d'application à la nature de Dieu}

Si l'on prétend que l'essentiel de la visée cherburienne, et cela sans doute s'est déterminé progressivement au fur et à mesure que Cherbury écrivait le De veritate, réside dans l'établissement des bases de la religion

14. $D V, 29 / 39$.

15. $D V, 57 / 77$.

16. $D V, 48 / 64$ : " [...] sed ideo communes esse perhibemus quia, nisi ipsi praecludamus viam, communes futurae sunt.» 
naturelle et de l'irénisme religieux qu'elle rend possible, on doit pouvoir appliquer la théorie cherburienne des niveaux de vérité à des notions théologiques. Il convient de préciser cependant que cet essai d'application est entièrement mon fait et ne se trouve pas chez Cherbury.

La veritas rei devrait donner à connaître ce qu'est Dieu en soi et non pour nous. En fait, les notions communes nous apprennent seulement qu'il est un et provident. Notre connaissance présente est bornée au temporel et au fini et ne nous permet pas de connaître Dieu tel qu'il est en lui-même. La veritas Dei demeure inconnue.

La veritas apparentiae, elle, pourrait porter sur la révélation qui correspond dans l'ordre religieux à ce qu'est le phénomène dans l'ordre des réalités concrètes et sensibles. La révélation est une indication faite par la divinité à quelqu'un pour lui montrer quelque chose de vrai ou de bon (elenchus verborum du De veritate). Il n'y a, pour Cherbury, de révélation qu'immédiate et en première personne (faite à moi-même, par exemple le signe du coup de tonnerre dans un ciel serein qui le décida à publier son $D e$ veritate). Toute révélation reçue d'autrui relève du témoignage historique, donc de la vérité de l'apparaître, et n'est plus à proprement parler révélation divine qui a pour marque distinctive, outre l'émotion qui l'accompagne, de «nous persuader quelque bien car c'est de là que l'on discerne les fausses d'avec les vraies ${ }^{17} \gg$. En un sens large, la vérité de l'apparaître en matière religieuse peut recouvrir aussi les multiples croyances détaillées dans le De religione gentilium.

La veritas conceptus recouvre toutes les théologies dogmatiques élaborées par les différents peuples et dont les contradictions externes et internes ont fait douter de la vérité de la chose correspondante.

La veritas intellectus, enfin, désigne l'ensemble des notions communes religieuses regroupées dans les cinq articles de la foi universelle.

Les conditions de conformité sont également détaillées par Cherbury, notamment en ce qui concerne les conditions d'acceptabilité de la révélation religieuse comme signe direct de la divinité adressé personnellement à quelqu'un pour le mouvoir à faire quelque bien.

Face à cette théorie, Popkin qui la résume rapidement accorde beaucoup d'importance aux critiques de Gassendi et de Descartes, ce qui se comprend bien, vu la perspective adoptée.

« Gassendi montrait que dès lors qu'avait été faite la distinction sceptique traditionnelle entre la vérité des choses en elles-mêmes et la vérité des apparences, le système de Herbert ne pouvait en rien contribuer à étendre notre savoir des apparences à la réalité. Nous n'avions conscience que de l'apparence des choses, que du goût sucré du miel et de la chaleur du feu. C'était

17. $D V, 226 / 290$. 
faire preuve d'une tournure d'esprit infortunée que d'essayer d'aller au-delà de ces apparences car seul Dieu connaissait la vraie nature des choses. Le système élaboré par le De veritate ne nous révélait pas la vérité dans toute sa pureté mais plutôt les conditions dans lesquelles la vérité nous apparaissait, c'est-àdire les conditions dans lesquelles nous parvenions à un savoir juste et utile dans le domaine de l'expérience; mais ce n'étaient pas là les conditions dans lesquelles nous allions découvrir l'inconditionnelle veritas rei ${ }^{18}$, "

Certes Popkin, après Gassendi et Descartes, n'a pas tort de souligner l'insuffisance du fondement métaphysique (par rapport à Dieu garant des vérités éternelles et des vérités tirées du cogito) ou du critère épistémologique du vrai chez Cherbury. Mais est-ce bien là son problème? Pas vraiment. Le vrai problème, c'est la liberté de penser en matière théologique. Descartes y avait d'ailleurs été sensible puisqu'il avoua à Mersenne avoir préféré la version française du De veritate (la traduction édulcorée de Mersenne) au texte latin qu'il n'avait fait que parcourir.

LA VISÉE CHERBURIENNE

La perspective de Cherbury n'est pas celle du savant ou du métaphysicien; c'est celle du laicus viator, du voyageur non docte poussé par un irrésistible et naturel besoin de bonheur infini, de béatitude et qui ne trouve pas dans les dogmatismes cléricaux et les anathèmes qui l'accompagnent de quoi contenter cette exigence. La religion du laï, puisque c'est l'expression idiomatique cherburienne pour désigner ce que nous appelons aujourd'hui religion naturelle, est la notion commune de la religion, laquelle est elle-même une notion commune « puisqu'il n'y a jamais eu siècle ou nation sans aucune religion ${ }^{19}{ }^{\prime}$. Qui dit laïc dit un homme qui n'est pas encombré par le credo d'une église ou le système d'une école; qui dit voyageur désigne un homme qui a le goût de la découverte et de l'aventure, qui ne craint pas d'explorer d'autres continents religieux, ceux de l'Antiquité d'abord, d'où cette somme que constitue le traité sur La Religion des payens, mais aussi ceux de pays proches (les musulmans) ou lointains pour y découvrir qu'à quelques détails près, il en va partout là-bas tout comme ici et qui, fort de cette conviction, n'hésite pas à forger un rite qui lui convienne. Qu'on se souvienne seulement que, chez lord Herbert, la prière traditiopnelle chrétienne n'était pas supprimée mais remplacée par

18. $H S$, p. 212.

19. $D V, 43 / 57$. 
une prière adressée à un Dieu providentiel, plein d'amour et de miséricorde, qui ne songe nullement à punir mais seulement à attirer l'homme vers lui dans une perspective qui évoque aussi bien la conversion néoplatonicienne que la vie surnaturelle chrétienne.

La réponse cherburienne à la «crise sceptique », pour reprendre l'expression de Popkin, n'est pas dans sa théorie de la vérité effectivement bien embrouillée et inefficace mais dans sa théorie des notions communes religieuses qui se donne le moyen de dégager l'invariant d'une variation (les cinq articles) permettant ensuite à chacun de construire la croyance et le culte dans lequel il se trouvera chez soi sans empêcher les autres d'en faire autant. La visée cherburienne n'est pas théorique mais pratique, pas spéculative mais politique au sens large : il s'agit de défendre, face au dogmatisme tant catholique que protestant, et de fonder, face à un scepticisme perçu comme délétère, les droits de la conscience individuelle, conscience vagabonde et musardière bien plus que conscience errante, conscience que ne traverse aucune angoisse, ni l'angoisse du péché ni le scandale du mal, et qui se prépare à la "fruition » béatifique éternelle sans renoncer à la jouissance des biens légitimes d'ici-bas.

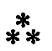

Herbert déplace donc la question de la vérité dans les sciences (qui était celle de Mersenne et de Gassendi) vers la question des voies personnelles d'un salut conçu comme contentement en cette vie et assurance de la béatitude éternelle dans l'autre. Ni sceptique chrétien ni fidéiste respectant la tradition ou une autorité surnaturelle, Herbert ne pose plus la question de la «vraie religion» en terme de vérité ou de fausseté mais en termes de « convivialité », du bien vivre ensemble. La vraie religion est celle où chacun se sent chez soi, celle donc qu'il habite et aménage à sa guise, celle qui favorise les rapports les plus souples et les plus harmonieux avec ses voisins sans mettre en péril la paix civile.

Lord Herbert n'a pas voulu élaborer une machine de guerre contre le scepticisme en dépit de ses affirmations péremptoires du début du De veritate: «Je n'ai point d'autre intention dans cette proposition (est veritas) que de dire qu'il y a de la vérité contre l'impertinence et la folie des sceptiques ${ }^{20}$. "Il y a de la vérité et Mersenne a fort bien fait de traduire ici par un partitif car, pour Cherbury, scepticisme et dogmatisme sont deux folies symétriques et connexes:

20. $D V, 8 / 10$. 
« [...] il y a eu dans tous les siècles des philosophes si malheureux et si opiniastres qu'ils ont enseigné avec autant de soin \& d'ardeur que l'on ne pouvait rien savoir comme ils ont enseigné que l'on pouvait connaître toutes choses. De là vient que les uns ont dit que la vérité était cachée dedans un puits qu'ils ne savaient qu'une chose à savoir qu'ils ne savaient rien \& cent autres sottises et contradictions semblables afin de paraitre les plus sages des hommes. Et d'ailleurs les autres ont dit avec un indicible préjudice de la vérité et avec une étrange arrogance que par les principes de la connaissance qui seuls nous appartiennent on pourrait trouver les principes de la constitution des choses afin de persuader qu'ils n'ignorent rien. C'est de là qu'ont commencé les différentes sectes, chacun cherchant à s'établir et à se mettre en réputation suivant la faveur et les suffrages du peuple ignorant ${ }^{21}$. 》

La lutte contre l'esprit de secte ne concerne pas seulement les écoles théologiques mais bien aussi les écoles philosophiques. Pour autant, son effort ne vise pas tant à fonder la science qu'à garantir la validité d'un savoir minimal, suffisant dans l'ordre religieux, indépendant de la foi en une révélation. À lui s'applique parfaitement le mot de Grotius, "Nobis modica theologia sufficit ». À la différence d'une théologie naturelle uniquement appuyée sur la raison (et faisant, par exemple, grand usage de la preuve a priori de l'existence de Dieu), il assure sa croyance sur un sentiment interne de Dieu. La diversité des opinions qui est une objection dirimante contre la prétention de l'instinct naturel à la vérité, pour un Gassendi qui veut à la fois atteindre un savoir objectif et laisser à la révélation, présentée et garantie par l'autorité et la tradition, le soin de donner les enseignements sur les fins dernières, n'est pas une objection pour Herbert puisque le salut passe par une multiplicité de chemins selon les temps et les lieux et peut donc s'accommoder d'une diversité d'opinions, particulièrement sur les modalités des moyens de salut.

Popkin voit dans le texte de Cherbury un essai avorté de réponse philosophique au scepticisme parce qu'il prend trop au sérieux l'entreprise du De veritate et qu'il néglige ce qui est tout de même, et rien qu'au nombre de pages, l'essentiel de l'œuvre de Herbert, à savoir les traités sur la religion. C'est aussi parce qu'il surévalue son aristotélisme et qu'il tait son inspiration stoïcienne. Cherbury est beaucoup plus proche de Sénèque et de Juste Lipse en ce domaine que d'Aristote ou des thomistes, ne serait-ce que par son refus d'une théologie rationnelle. Sans en adopter les principes et les requêtes, il retrouve en un sens une pratique sceptique puisqu'il accomplit le programme de Sextus Empiricus :

21. $D V, 2 / 2-3$. 
«Prenant toujours la vie pour guide, nous affirmons sans dogmatisme que les dieux existent, que nous vénérons les dieux et que nous leur vouons reconnaissance $^{22}$. »

La réponse cherburienne au scepticisme, ce n'est pas le rejet du phénoménisme mais un perspectivisme pratique qui recherche non la religion la plus vraie mais la meilleure puisqu'il y a du vrai et du bon en toutes $-\mathrm{y}$ compris dans l'Islam - la religion qui permet le mieux de vivre en paix. L'échec des zététiques cherburiennes, c'est bien l'échec d'une solution épistémologique à la crise sceptique mais la postérité des cinq articles fondamentaux de Cherbury (les sept dogmes fondamentaux du Traité théologico-politique de Spinoza, le credo minimum de Locke...) signifie aussi la réussite, momentanée mais historiquement significative, d'une solution à la crise de la théologie dogmatique par la promotion d'une religion réduite à son noyau éthique.

Jacqueline LAGRÉE (janvier 1997).

22. Sextus EmpiRicus, Hypotyposes pyrrhoniennes, liv. III, chap. II, trad. Jean-Paul Dumont, in Les Sceptiques grecs, Paris, Presses universitaires de France, 1966, p. 185. 